\title{
Modification of the method to establish a hepatic VX2 carcinoma model in rabbits
}

\author{
HUAN TONG $^{1,2}$, LI-GENG DUAN ${ }^{3}$, HONG-YING ZHOU ${ }^{1}$ and SHI FENG ${ }^{1}$ \\ ${ }^{1}$ Department of Human Anatomy, West China School of Basic Medical Sciences and Forensic Medicine; \\ Departments of ${ }^{2}$ Gastroenterology and ${ }^{3}$ Emergency, West China Hospital, \\ Sichuan University, Chengdu, Sichuan 610041, P.R. China
}

Received January 23, 2017; Accepted December 15, 2017

DOI: $10.3892 / \mathrm{ol} .2018 .7980$

\begin{abstract}
The hepatic VX2 carcinoma model in rabbits is widely used for the preclinical study of hepatocellular carcinoma. In the present study, a modification was made to the conventional method to establish the animal model, as the conventional method gives rise to frequent tumor seeding due to the drop-out of tumor fragments. In order to evaluate each distinct method of establishing the model, the rabbits were divided into two groups: Group A (the conventional method; $\mathrm{n}=20$ ) and group B (the modified method; $\mathrm{n}=20$ ). All surgical details were recorded for reference. At 14 days post-surgery, contrast-enhanced computed tomography (CECT) and autopsy were conducted. Microscopic morphology of tumor cells was observed using hematoxylin and eosin (H\&E) and transmission electron microscopy (TEM). Vascular endothelial grow th factor (VEGF) and cluster of differentiation (CD)31 were detected via immunochemistry and reverse transcription-polymerase chain reaction. In total, 19 rabbits in each group succeeded in model establishment. Throughout the surgery, group A experienced a longer surgery time compared with group B (group A vs. group B, $22.57 \pm 1.34$ vs. $20.17 \pm 1.50 \mathrm{~min} ; \mathrm{P}<0.001)$, an increased tumor fragment drop-out frequency (group A vs. group B, $1.84 \pm 0.96$ vs. $1.16 \pm 0.38 ; \mathrm{P}=0.008)$ and an increased peritoneal nodule incidence (group A vs. group B, 35 vs. 5\%, P=0.042).
\end{abstract}

Correspondence to: Dr Hong-Ying Zhou or Dr Shi Feng, Department of Human Anatomy, West China School of Basic Medical Sciences and Forensic Medicine, Sichuan University, 3rd Section, 17 South Renmin Road, Chengdu, Sichuan 610041, P.R. China

E-mail: eaglezhyxzy@163.com

E-mail: jetmork@126.com

Abbreviations: CD31, cluster of differentiation 31; CECT, contrast-enhanced computed tomography; HCC, hepatocellular carcinoma; H\&E, hematoxylin and eosin; IOD, integrated optical density; RT-PCR, reverse transcription-polymerase chain reaction; TACE, transarterial chemoembolization; TEM, transmission electron microscope

Key words: liver neoplasm, animal model, method modification, VX2 carcinoma, gelatin sponge
As for CECT, H\&E and TEM, hepatic VX2 allografts in the two groups demonstrated similar imaging presentations and tumor cell morphology. In addition, VEGF and CD31 levels did not differ between the two groups. In conclusion, the modified method for the establishment of hepatic VX2 carcinoma model in rabbits may decrease tumor fragment drop-out frequency during surgery and incidence of tumor seeding without affecting the properties of VX2 carcinoma.

\section{Introduction}

Hepatocellular carcinoma (HCC) accounts for $\sim 700,000$ mortalities worldwide annually, being the third leading cause of cancer-associated mortality (1). As it is difficult to diagnose at an early stage, HCC is usually concurrent with other chronic liver diseases, and the majority of patients with HCC are diagnosed at an intermediate or advanced stage; such patients are not viable for curative surgery, leading to poor prognoses (2). Transarterial chemoembolization (TACE), which embolizes the artery supplying the tumor and administers chemotherapeutic agents precisely, has been applied to treat HCC for decades (3). However, TACE seldom achieves complete tumor necrosis and the resultant secondary hypoxia may induce angiogenesis, limiting the efficacy of TACE (4). Therefore, further research on TACE, as well as a widely used and stable animal liver tumor model, is required.

The VX2 carcinoma model was firstly developed in the 1930s and can be implanted in various areas, including the liver, kidney and lung; however, this model can only be established in rabbits (5). The main blood supply from the hepatic artery and the rapid growth demonstrated by the hepatic VX2 carcinoma model are similar to the characteristics of human HCC (6). Additionally, rabbits are large enough for catheterization, as opposed to other animal models such as mice and rats, which explains why this model is widely used by interventional radiologists for studying HCC (7).

The establishment of a hepatic VX2 carcinoma model requires surgical skills. Surgical implantation of the tumor fragment into the liver is a common conventional method; however the risk of tumor leakage and atopic seeding cannot be avoided, and negatively impacts sequential evaluation of the efficacy of this therapeutic modality in treating VX2 carcinoma (8). Therefore, it is necessary to investigate adequate 
modifications to the conventional method, and the present study was conducted with the aim of comparing the efficacy of the conventional method with that of a new and modified approach.

\section{Materials and methods}

Animal model establishment. The animal experimental protocol was approved by the Institutional Animal Care and Use Committee of Sichuan University (Chengdu, China), and was conducted in accordance with animal experimental guidelines set by Sichuan University. A total of 40 12-month-old male New Zealand white rabbits, weighing approximately 2.3-2.5 kg, were purchased from the Experimental Animal Center of West China Medical Center (Chengdu, China). Two male VX2 carrier rabbits weighing 2.3-2.5 kg were obtained from Union Hospital, Huazhong University of Science and Technology (Wuhan, China). All the rabbits used in the present study were kept under a 12-h light-dark cycle at $26^{\circ} \mathrm{C}$, $0.03 \%$ carbon dioxide and one atmosphere with free access to food and water prior to preoperative fasting. The humane endpoints were cachexia, dyspnea and grooming inability.

The 40 rabbits were randomly assigned into two groups in order to establish the hepatic VX2 carcinoma model via two distinct methods. The rabbits were not permitted any food or water 12 and $6 \mathrm{~h}$ prior to surgery, respectively. Tumor bulks were excised from the VX2 carrier rabbit, minced into small fragments with a diameter of 1-2 mm, and stored in Dulbecco's Modified Eagle Medium (DMEM; Thermo Fisher Scientific, Inc., Waltham, MA, USA). Tumor fragments were prepared according to the aforementioned method prior to tumor implantation. Following adequate general anesthesia using sodium pentobarbital $(30 \mathrm{mg} / \mathrm{kg}$ ) administered intravenously via the marginal ear vein, and a small surgical cut $(3.05 \pm 0.21 \mathrm{~cm}$ in group A, $3.08 \pm 0.25 \mathrm{~cm}$ in group B) was made in the subxiphoid area under aseptic conditions. Subsequently, the left lobe of the liver was exposed and a 5-mm deep tunnel was made on the surface.

The sequential step differed between the two groups. In group A (the conventional method), a tumor fragment was implanted into the tunnel, followed by blocking of the tunnel with gelatin sponge (Fig. 1). In group B (the modified method), the tumor fragment was embedded in gelatin sponge prior to implantation into the tunnel as a whole, followed by gelatin sponge to block the tunnel (Fig. 1).

Following tumor implantation, the exposed liver lobe was returned into the abdominal cavity and the abdomen wall was closed by suture; all surgical details were recorded. Rabbits were administered penicillin G (100,000 U) twice daily for 3 days following tumor implantation.

Contrast-enhanced computed tomography (CECT). VX2 carcinoma usually exhibits optimal tumor viability without significant necrosis 14 days after tumor fragment implantation (9). Therefore, all rabbits in the present study underwent CECT 14 days after surgery to assure the formation of the tumor nodule in the liver.

Rabbits were not permitted food or water 12 and $6 \mathrm{~h}$ prior to CECT, respectively. CECT was performed using a Philips Brilliance 64 CT Scanner (Philips Healthcare, Amsterdam,
Netherlands). Following general anesthetizing and correct positioning of the rabbits, images were obtained of the plain, arterial and venous phase, sequentially. Arterial and venous phases were obtained 10 and $30 \mathrm{sec}$ after the contrast injection (injection speed $0.5 \mathrm{ml} / \mathrm{s}$, injection volume $5 \mathrm{ml}$ ), respectively. The parameters for CECT were: Voltage, $120 \mathrm{kV}$; current, $106 \mathrm{~mA}$; thickness, $3 \mathrm{~mm}$; pitch 0.891; and matrix, 512x512.

Macroscopic and microscopic evaluation. Rabbits were sacrificed following CECT. The mass, largest tumor diameter, and the number of tumor foci in the liver were measured, and the number of peritoneal nodules were recorded.

The hepatic allograft was retained for analysis via light microscopy and transmission electron microscopy. Briefly, tumor tissues were fixed in $4 \%$ paraformaldehyde for $24 \mathrm{~h}$ at room temperature and embedded in paraffin. Then, they were sliced into $5-\mu \mathrm{m}$ thick sections and stained with hematoxylin for $1 \mathrm{~min}$ and eosin for $1 \mathrm{~min}(\mathrm{H} \& \mathrm{E})$ at room temperature for further observation under a light microscope (CX31; Olympus Corporation, Tokyo, Japan).

In addition, for electron microscopy, the tumor specimens were fixed in $4 \%$ glutaraldehyde and $1 \%$ osmium tetroxide, and then embedded in EMbed 812 following dehydration. Ultra-thin sections (60-nm thick) were examined using a transmission electron microscope (TEM; 600IV; Hitachi, Ltd., Tokyo, Japan) following double staining with $2 \%$ uranyl acetate for $30 \mathrm{~min}$ and $3 \%$ lead citrate for $10 \mathrm{~min}$ at room temperature (magnification, x6,000). The morphology of tumor cells was observed.

Immunohistochemistry for vascular endothelial growth factor (VEGF) and cluster of differentiation (CD)31. The 5- $\mu \mathrm{m}$ thick paraffin-embedded tissue sections on poly-L-lysine coated glass slides were treated at $60^{\circ} \mathrm{C}$ for $20 \mathrm{~min}$, then deparaffinized in xylene for $25 \mathrm{~min}$ and rehydrated through a series of descending ethanol gradients $(100 \%$ ethanol for $3 \mathrm{~min}$, $100 \%$ ethanol for $3 \mathrm{~min}, 95 \%$ ethanol for $3 \mathrm{~min}, 90 \%$ ethanol for $3 \mathrm{~min}$, sequentially) at room temperature. For non-specific blocking, 10\% goat serum (cat. no. SP-9002; Zymed; Thermo Fisher Scientific, Inc.) was added and incubated for $15 \mathrm{~min}$ at room temperature. Subsequently, sections were incubated with a mouse anti-rabbit VEGF antibody (cat. no. ab28775; Abcam, Cambridge, UK) and a mouse anti-rabbit CD31 antibody (cat. no. 08-1425; Zymed; Thermo Fisher Scientific, Inc.) at a 1:250 dilution at $4^{\circ} \mathrm{C}$ overnight. A positive reaction was revealed using the streptavidin-biotin-peroxidase (ready-to-use; cat. no. SP-9002; Zymed; Thermo Fisher Scientific, Inc.) technique at room temperature for 10-15 min. Sections were then incubated with 3,3'-diaminobenzidine and counterstained with Meyer's hematoxylin (cat. no. 03791; Sigma-Aldrich; Merck KGaA, Darmstadt, Germany) at room temperature for $30 \mathrm{sec}$. Image-Pro Plus 6.0 software (Media Cybernetics, Inc., Rockville, MD, USA) was used to score the integrated optical density (IOD) from VEGF expression in tumor cells, and to count the number of CD31-positive cells per visual field under light microscope (magnification, x200; CX31; Olympus Corporation). Each value was the mean from 10 visual fields.

Reverse transcription-polymerase chain reaction (RT-PCR) for VEGF. Total RNA was extracted from tumor tissues using TRIzol ${ }^{\circledR}$ reagent (cat. no. 15596-026, Invitrogen; Thermo Fisher 

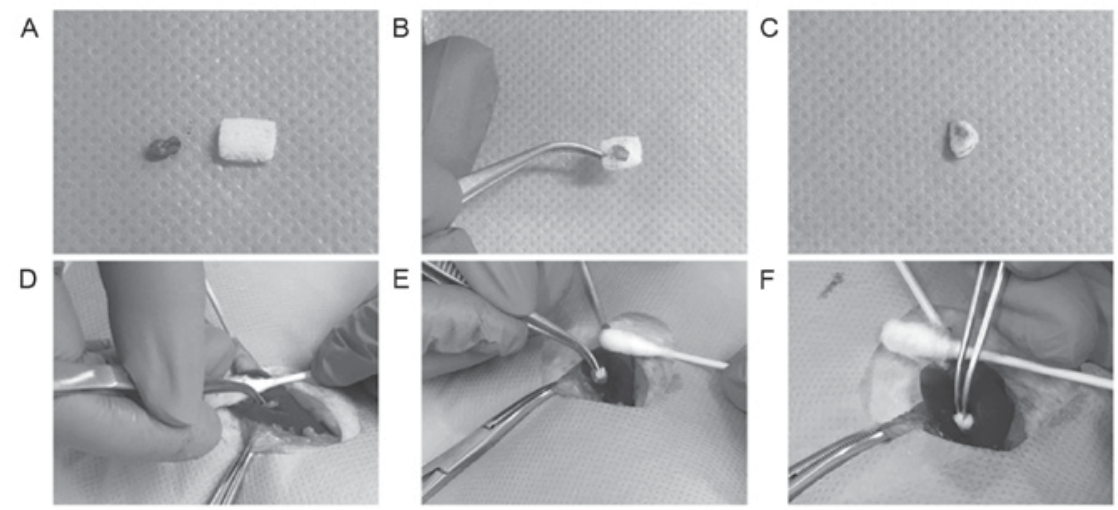

Figure 1. Key steps for model establishment. (A) A tumor fragment and a piece of gelatin sponge. (B) Tumor fragment being embedded in the gelatin sponge. (C) Tumor fragment embedded in the gelatin sponge. (D) The tumor fragment is implanted into the liver of a rabbit from group A. (E) The tumor fragment embedded in gelatin sponge is implanted into the liver of a rabbit from group B. (F) A piece of gelatin sponge is applied to block the tunnel in the liver.

Scientific, Inc.). Quantification and purity of the extracted RNA were conducted and tested using a spectrophotometer (GeneQuant 1300; Biochrom, Ltd., Cambridge, UK). RT-PCR was conducted using a thermal cycler (PTC-100; Bio-Rad Laboratories, Inc., Hercules, CA, USA) and ReverAid ${ }^{\text {тм }}$ First Strand cDNA Synthesis kit (cat. no. Fermentas; Thermo Fisher Scientific, Inc.), according to the manufacturer's protocol.

The primer sequences for the sense and antisense chain of GAPDH were 5'-TCTCGTCCTCCTCTGGTGCTCT-3' and 5'-AAGTGGGGTGATGCTGGTGC-3', respectively. The primer sequences for the sense and antisense chain of VEGF were 5'-ATGGCAGAAGAAGGAGAC-3' and 5'-ATT TGTTGTGCTGTAGGAAG-3', respectively. The PCR cycle profile was $94^{\circ} \mathrm{C}$ for $30 \mathrm{sec}, 52^{\circ} \mathrm{C}$ for $60 \mathrm{sec}$ and $72^{\circ} \mathrm{C}$ for $60 \mathrm{sec}$, for 30 cycles. The amplification was terminated by a final extension step at $72^{\circ} \mathrm{C}$ for $2 \mathrm{~min}$. An internal control (GAPDH) was amplified at the same time. RT-PCR products were quantified by running them on a $1 \%$ agarose gel with ethidium bromide, and then by scanning the gel with an imaging system (Gel Doc 2000; Bio-Rad Laboratories, Inc.). The data were normalized as a ratio of gray scale IOD of the objective band over GAPDH, and using Quantity One v4.4.0.36 software (Bio-Rad Laboratories, Inc.) for densitometry.

Statistical analysis. Quantitative data are expressed as the mean \pm standard deviation and were calculated using the Student's t-test. Qualitative data are expressed as a percentage and were calculated using the $\chi^{2}$ test. All statistical analysis was performed using SPSS v.19.0 for Windows (IBM Corp., Armonk, NY, USA). P<0.05 was considered to indicate a statistically significant difference. The correlation between two variables was determined by Pearson's correlation analysis or by Spearman's rank correlation analysis, depending on the type of data.

\section{Results}

Feasibility of each distinct method for establishing the hepatic VX2 carcinoma model. A total of 40 rabbits split equally into two groups underwent two distinct approaches to establish the hepatic VX2 carcinoma model. Of the 20 rabbits, 19 rabbits in each group succeeded in model establishment. One rabbit died in each group following surgery. Throughout the surgical procedure, group A received comparable incision lengths to group B; however, group A experienced longer surgery times compared with group B. During surgery, group A experienced an increased tumor fragment drop-out frequency compared with group B (Table I). Linear correlation analysis using Pearson's correlation indicated a statistically significant correlation between the tumor fragment drop-out frequency and the surgery time $(\mathrm{r}=0.479, \mathrm{P}=0.002)$.

CECT manifestations and autopsy results. CECT demonstrated the allograft in the left lobe of the liver among successful model established rabbits. These nodules of diameters between 2.0 and $3.0 \mathrm{~cm}$ were enhanced in the arterial phase following contrast injection and became faint in the venous phase (Fig. 2).

Although the distinct methods were applied to establish the hepatic VX2 carcinoma model, no statistically significant differences were identified between the two groups regarding the tumor foci number, tumor foci mass and diameter of the largest tumor focus in the liver (Table I). Furthermore, the peritoneal nodule incidence in group A was six times higher than that of group B. Spearman's rank correlation analysis revealed a statistically significant correlation between the tumor fragment drop-out frequency and the incidence of peritoneal nodules $\left(r_{s}=0.747, P<0.001\right)$. No lung metastases were observed in groups $\mathrm{A}$ or $\mathrm{B}$.

$H \& E$ staining and TEM. H\&E-positive tumor cells in groups $\mathrm{A}$ and $\mathrm{B}$ exhibited characteristics of atypia, hyperchromatosis and numerous mitoses under light microscopy; these cells were arranged in clusters (Fig. 3). Furthermore, using TEM, an irregular shape of the nuclear membrane and nucleolus, and an extension of the endoplasmic reticulum were observed in the two groups (Fig. 3).

VEGF and CD31 expression in the liver allografts. Liver allografts in group A and group B demonstrated that $70-80 \%$ tumor cells were VEGF positive by immunohistochemical staining, primarily located in the cytoplasm of tumor cells (Fig. 3). VEGF expression levels, revealed by 
Table I. Feasibility of model establishment methods and autopsy results.

\begin{tabular}{lccr} 
Characteristic & method $(\mathrm{n}=20)$ & method $(\mathrm{n}=20)$ & P-value \\
\hline Operation time, min $^{\mathrm{a}}$ & $22.57 \pm 1.34$ & $20.17 \pm 1.50$ & $<0.01$ \\
Incision length, $\mathrm{cm}$ & $3.05 \pm 0.21$ & $3.08 \pm 0.25$ & 0.57 \\
Tumor fragment drop-out frequency & $1.16 \pm 0.38$ & $<0.01$ \\
Tumor foci in the liver & $1.84 \pm 0.96$ & & 0.80 \\
$\mathrm{~N}$ & & & 0.86 \\
Mass, $\mathrm{g}$ & $1.37 \pm 0.68$ & $1.32 \pm 0.58$ & 0.79 \\
Largest diameter, cm & $18.27 \pm 5.93$ & $18.60 \pm 5.49$ & 0.04 \\
Abdominal implantation, $\mathrm{n}^{\mathrm{a}}$ & $2.51 \pm 0.27$ & $2.53 \pm 0.24$ & 1.00 \\
Lung metastases, $\mathrm{n}$ & 7 & 1 & 0 \\
\hline
\end{tabular}

${ }^{a} \mathrm{P}<0.05$. Data are presented as the mean \pm standard deviation.

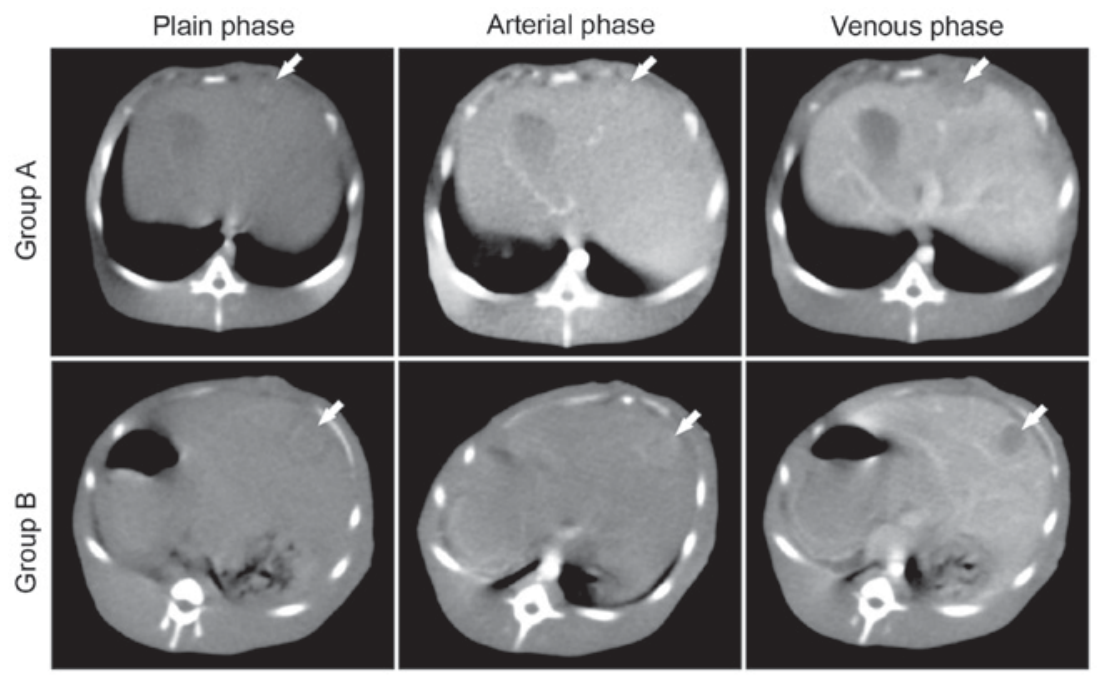

Figure 2. Contrast-enhanced computed tomography imaging of rabbits in groups A and B. The white arrows indicate the hepatic VX2 carcinoma foci in the liver.

immunohistochemistry, were comparable in the two groups [IOD for group A, $(3.76 \pm 1.25) \times 10^{5}$; group B, (3.54 \pm 1.04$) \times 10^{5}$; $\mathrm{P}=0.566]$. The levels of VEGF mRNA investigated by RT-PCR in the two groups were also comparable (IOD ratio of VEGF over GAPDH for group A, 1.57 \pm 0.40 ; group B, $1.63 \pm 0.41$; $\mathrm{P}=0.647$ ) (Fig. 3).

In addition, the hepatic VX2 allografts in groups A and B were rich in microvessels; this was revealed by the comparable levels of CD31-positive cells (group A, 21.35 \pm 4.97 ; group B, 23.48 $\pm 5.06 ; \mathrm{P}=0.200$ ) (Fig. 3).

\section{Discussion}

As a prevailing animal model in the study of interventional treatment for $\mathrm{HCC}$, the hepatic VX2 carcinoma model is established through the surgical implantation of tumor fragments, or via the injection of tumor suspension cells. Notably, the injection of tumor suspension cells is more likely to result in leakage and seeding of tumor cells, and has a decreased rate of successful model establishment, compared with the surgical implantation of tumor fragments $(10,11)$. Conversely,
Virmani et al (10) suggested that the injection of tumor suspension cells was recommendable. Nevertheless, the difficulties in determining the homogeneity of tumor suspension cell count, variations in the cell amount, and injection into small vessels of the liver result in a variation in tumor size and foci number, were also identified by Virmani et al (10).

In terms of hepatic VX2 carcinoma model establishment, conventional surgical implantation of tumor fragments presents a higher success rate and a lower frequency of metastasis in comparison with the injection of tumor suspension cells (10). In the present study, the sizes of the hepatic allograft in each group were comparable, and the mean number of hepatic foci was 1.3-1.4, overcoming the aforementioned disadvantages of tumor suspension cell injection. Therefore, surgical implantation of tumor fragments is superior to injection of tumor suspension cells in establishing a hepatic VX2 carcinoma model.

However, it is noteworthy that animals in group A, in which tumor fragments were implanted by the conventional method, experienced an increased incidence of peritoneal nodules $(36.8 \%)$ compared with group B. Peritoneal nodules 
A

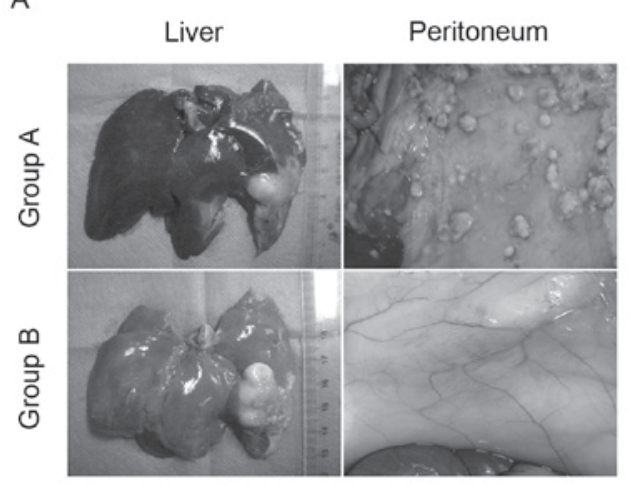

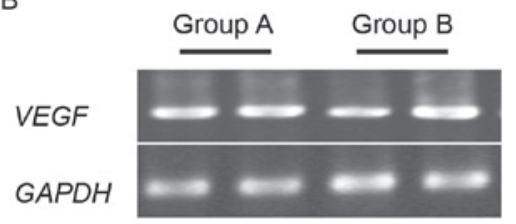

C

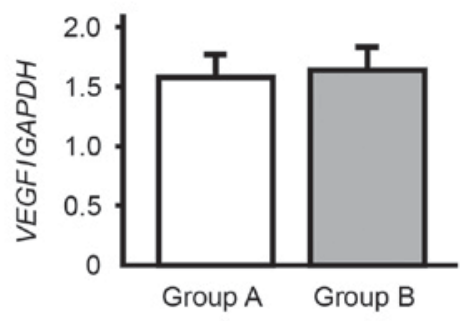

D

$H \& E$

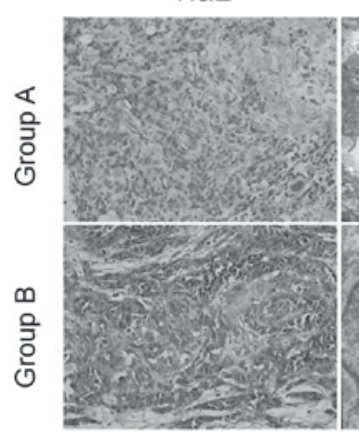

TEM

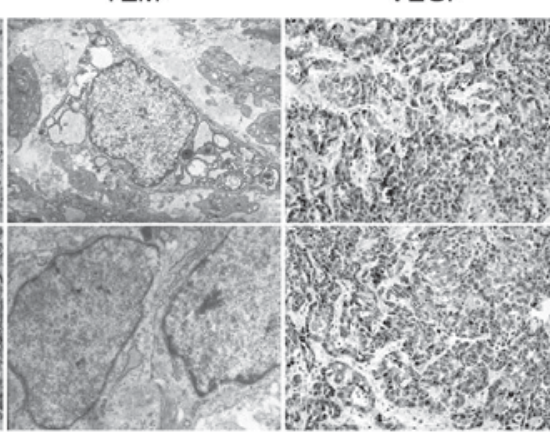

CD 31

Figure 3. Autopsy findings, and microscopic manifestations and angiogenesis of hepatic VX2 allografts. (A) Comparable tumor size of hepatic VX2 allografts in groups A and B; however, there was significant peritoneal implantation in group A, compared with in group B. (B) Expression of VEGF mRNA and GAPDH mRNA in hepatic VX2 allografts of groups A and B, as determined by RT-PCR. (C) Integrated optical density ratio of VEGF over GAPDH, as determined by RT-PCR, in hepatic VX2 allografts of groups A and B. (D) There were similar manifestations revealed by H\&E staining (magnification, x400) and TEM (magnification, x6,000), and similar expression levels of VEGF and CD 31, as determined by immunohistochemistry, (magnification, x400) in the hepatic VX2 allografts of both groups. CD, cluster of differentiation; GAPDH, glyceraldehyde-3-phosphate dehydrogenase; H\&E, hematoxylin and eosin; RT-PCR, reverse transcription-polymerase chain reaction; TEM, transmission electron microscopy; VEGF, vascular endothelial growth factor.

may be attributed to the high invasive and metastatic properties of VX2 carcinoma $(12,13)$. Previous studies have demonstrated that the majority of rabbits underwent metastasis $\sim 40$ days after tumor implantation $(8,14)$. Distal metastasis and abdominal implantation occurring at a late stage of liver carcinoma were usually accompanied by either intrahepatic metastasis or large primary implantation foci (8). Conversely, in the present study, rabbits in group A, whose peritoneum was scattered with nodules, possessed hepatic allograft diameters of $<5 \mathrm{~cm}$ at 14 days after tumor implantation, without concomitant intrahepatic or lung metastasis. This result cannot be explained by the high invasive and metastatic properties of VX2 carcinoma, but may possibly occur as a result of tumor cell seeding.

Similar to the results of previous studies, the occurrence of abdominal implantation in group A (the conventional method) implied that abdominal implantation was a common problem for rabbit hepatic VX2 carcinoma models $(9,15)$. A previous study attempted to implant tumor fragments into liver with a syringe; this method resulted in a decreased incidence of abdominal implantation from $>30.0$ to $13.3 \%$ (15). Previously, we evaluated the aforementioned syringe method and identified that it is not practical, as tumor fragments are difficult to inject through the tip of the syringe and easily cause obstructions (unpublished data); therefore, it was not utilized in the present study. By contrast, the modified method using gelatin sponge in the present study had an abdominal implantation incidence of only $5.3 \%$, demonstrating its advantages compared with the syringe method (13.3\%) (15).

During the conventional surgical implantation of tumor fragments in the present study, the friction experienced between the tumor fragment and the forceps, and between the tumor fragment and the liver, was insufficient to firmly hold the tumor fragment in the liver. As a result, the tumor fragment was occasionally dropped during the surgery, which potentially increased the possibility of tumor cell seeding, as well as prolonging the duration of surgery. Additionally, once tumor bulks were properly minced into fragments in DMEM, the surface of the tumor fragments may contain a small amount of fluid with free tumor cells, increasing the possibility of tumor cell seeding during tumor implantation. The correlation analysis of the tumor fragment drop-out frequency and the incidence of peritoneal nodules supports the hypothesis that peritoneal nodules originate from tumor cell seeding during surgery.

The modified method in the present study embedded the tumor fragment into gelatin sponge, which provided a physical barrier and increased the friction between the tumor fragment and the liver, decreasing the drop-out frequency of the tumor fragment during surgery. Therefore, abdominal implantation was identified in only $5.3 \%$ of rabbits in group B. 
However, as an embolizing material, the segregation function of the gelatin sponge may inhibit tumor growth, potentially through insufficient blood and nutrient supply (16), although it significantly decreased the incidence of peritoneal nodules in the present study. To the contrary, the present study demonstrated that there was no significant distinction between the conventional and the modified methods with regard to the number, mass and largest diameter of tumor foci in the liver. The gelatin sponge has previously been used as an embolizing material in TACE; however, it is seldom used nowadays for its temporary embolizing effect, as it is a loose and absorbable material (16). Therefore, in the modified method, the gelatin sponge served the function of preventing tumor fragment drop-out and tumor cell implantation without completely restricting the supply of oxygen and nutrients to the tumor fragments.

There is an additional concern, regarding whether the inclusion of gelatin sponge influences vessel formation at 14 days after surgery. VEGF and CD31 expression was measured in the present study. VEGF is an important and potent pro-angiogenesis factor, and CD31 represents the microvessel density in tissue $(17,18)$. The present study did not identify any significant differences in VEGF or CD31 levels between the two groups. Furthermore, CECT imaging of the two groups was consistent with the classic presentation of HCC (19). Hence, the inclusion of embedding sponge gelatin in the modified method does not appear to affect the blood supply of hepatic VX2 carcinoma at 14 days after surgery.

In conclusion, the modified method for the establishment of hepatic VX2 carcinoma model in rabbits may decrease the tumor fragment drop-out frequency during surgery and the incidence of tumor seeding without affecting the properties of VX2 carcinoma. It is, therefore, recommended for use in the establishment of the hepatic VX2 carcinoma model in rabbits.

\section{References}

1. Ferlay J, Soerjomataram I, Dikshit R, Eser S, Mathers C, Rebelo M, Parkin DM, Forman D and Bray F: Cancer incidence and mortality worldwide: Sources, methods and major patterns in GLOBOCAN 2012. Int J Cancer 136: E359-E386, 2015.

2. Villanueva A, Hernandez-Gea V and Llovet JM: Medical therapies for hepatocellular carcinoma: A critical view of the evidence. Nat Rev Gastroenterol Hepatol 10: 34-42, 2012.

3. Crocetti L, Bargellini I and Cioni R: Loco-regional treatment of HCC: Current status. Clin Radiol 72: 626-635, 2017.

4. Matsui O: Current status of hepatocellular carcinoma treatment in Japan: Transarterial chemoembolization. Clin Drug Invest 32 (Suppl 2): S3-S13, 2012.
5. Kidd JG and Rous P: Cancers deriving from the virus papillomas of wild rabbits under natural conditions. J Exp Med 71: 469-494, 1940.

6. Ramirez LH, Julièron M, Bonnay M, Koscielny S, Zhao Z, Gouyette A and Munck JN: Stimulation of tumor growth in vitro and in vivo by suramin on the VX2 model. Invest New Drugs 13: 51-53, 1995

7. Geschwind JF, Artemov D, Abraham S, Omdal D, Huncharek MS McGee C, Arepally A, Lambert D, Venbrux AC and Lund GB: Chemoembolization of liver tumor in a rabbit model: Assessment of tumor cell death with diffusion weighted MR imaging and histologic analysis. J Vasc Interv Radiol 11: 1245-1255, 2000.

8. Tong H, Li X, Zhang CL, Gao JH, Wen SL, Huang ZY, Wen FQ, $\mathrm{Fu}$ P and Tang CW: Transcatheter arterial embolization followed by octreotide and celecoxib synergistically prolongs survival of rabbits with hepatic VX2 allografts. J Dig Dis 14: 29-37, 2013.

9. Guan L: Angiogenesis dependent characteristics of tumor observed on rabbit VX2 hepatic carcinoma. Int J Clinical Exp Pathol 8: 12014-12027, 2015.

10. Virmani S, Harris KR, Szolc-Kowalska B, Paunesku T, Woloschak GE, Lee FT, Lewandowski RJ, Sato KT, Ryu RK, Salem R, et al: Comparison of two different methods for inoculating VX2 tumors in rabbit livers and hind limbs. J Vasc Interv Radiol 19: 931-936, 2008

11. Sun JH, Zhang YL, Nie CH, Yu XB, Xie HY, Zhou L and Zheng SS: Considerations for two inoculation methods of rabbit hepatic tumors: Pathology and image features. Exp Ther Med 3: 386-390, 2012

12. Parvinian A, Casadaban LC and Gaba RC: Development, growth, propagation and angiographic utilization of the rabbit VX2 model of liver cancer: A pictorial primer and 'how to' guide. Diagn Interv Radiol 20: 335-340, 2014.

13. White SB, Chen J, Gordon AC, Harris KR, Nicolai JR, West DL and Larson AC: Percutaneous ultrasound guided implantation of VX2 for creation of a rabbit hepatic tumor model. PLoS One 10: e0123888, 2015

14. Tong H, Li X, Zhang CL, Gao JH, Wen SL, Huang ZY and Tang CW: Octreotide and celecoxib synergistically encapsulate VX2 hepatic allografts following transcatheter arterial embolisation. Exp Ther Med 5: 777-782, 2013.

15. Chen Z, Kang Z, Xiao EH, Tong M, Xiao YD and Li HB: Comparison of two different laparotomy methods for modeling rabbit VX2 hepatocarcinoma. World J Gastroenterol 21: 4875-4882, 2015

16. Osuga K, Maeda N, Higashihara H, Hori S, Nakazawa T, Tanaka K, Nakamura M, Kishimoto K, Ono Y and Tomiyama N: Current status of embolic agents for liver tumor embolization. Int J Clin Oncol 17: 306-315, 2012.

17. Lokmic Z and Mitchell GM: Visualisation and stereological assessment of blood and lymphatic vessels. Histol Histopathol 26: 781-796, 2011.

18. Bupathi M, Kaseb A, Meric-Bernstam F and Naing A: Hepatocellular carcinoma: Where there is unmet need. Mol Oncol 9: 1501-1509, 2015

19. Zhang W, Lai SL, Chen J, Xie D, Wu FX, Jin GQ and Su DK: Validated preoperative computed tomography risk estimation for postoperative hepatocellular carcinoma recurrence. World J Gastroenterol 23: 6467-6473, 2017. 